\title{
EMPLEABILIDAD EN EL SECTOR TURÍSTICO: EL CASO DE LOS EGRESADOS EN TURISMO DE LA UNIVERSIDAD DE SEVILLA
}

\author{
María Isabel Fresneda Rocamora ${ }^{1}$ \\ Recepcionista en Hotel Silken Al-Andalus Palace Sevilla \\ maribelfresneda91@gmail.com
}

(Fecha envío: 07/10/18 - Fecha aceptación: 30/11/18)

\section{Resumen}

El presente trabajo de fin de grado persigue identificar cuáles son las competencias específicas y transversales que el mercado laboral turístico está exigiendo a los profesionales en la actualidad. Para ello, se va a proceder a explicar y analizar desde una visión crítica la evolución de los estudios universitarios en turismo en las universidades españolas y, en particular, en la Universidad de Sevilla, con el objetivo de averiguar hasta qué punto esta formación se adapta a las verdaderas exigencias de las empresas del sector.

Tras una revisión de la evolución de los estudios universitarios en Turismo en España y en la Universidad de Sevilla, así como de la empleabilidad existente en el sector, se ha procedido a realizar un exhaustivo trabajo de campo entre los empresarios turísticos, que arrojarán resultados importantes acerca de aquello que se exige a los egresados en turismo en el momento de incorporarse al mercado laboral.

\section{Palabras clave}

turismo, formación universitaria, empleabilidad, competencias transversales, competencias específicas

\section{Abstract}

The present final degree project aims to identify which are the specific and transversal competences that the tourism labour market is demanding from professionals at these days. To do this, we will proceed to explain and analyse from a critical point of view the evolution of university studies in tourism at Spanish universities and at the University of Seville, with the aim of finding out to what extent this training is adapted to the real demands of companies in the sector.

After a review of the evolution of the university studies in Tourism in Spain and the University of Seville, as well as the employability existing in the sector, an exhaustive fieldwork has been carried out among tourist entrepreneurs, which will yield important results about what is required of graduates in tourism at the time of joining the labour market.

\section{Keywords:}

tourism, university education, employability, transversal competences, specific competence

\section{INTRODUCCIÓN}

\subsection{Objetivo del estudio}

El objetivo principal de esta investigación es averiguar, ante todo, cuáles son las variables que afectan a la contratación de los egresados en turismo, si son exógenas y debemos culpar de todo esto a la enorme crisis en la que se ha visto sumergida España, o endógenas, siendo el fallo principal la formación impartida al estudiante.

De este modo se hace más que necesario estudiar cómo ha evolucionado la formación desde el momento en que se reconoce la importancia del turismo como actividad económica en España hasta la actualidad, comprobar si el plan de estudios desde el 1996 ha sufrido modificaciones y analizar si estas han sido negativas o positivas para, de este modo, proceder a profundizar sobre cuál es la situación del mercado laboral actual, así como si hay puestos de trabajo en el sector acorde a la formación de los egresados.

\footnotetext{
${ }^{1}$ Grado en Turismo, Universidad de Sevilla.
} 
En otras palabras, a lo largo del texto aquí desarrollado se van a estudiar y averiguar las exigencias que los empresarios tienen respecto a los egresados del grado de turismo, así como analizar el nivel de preparación de estos para la verdadera lucha, ese campo de batalla que algunos Ilaman "Mercado laboral".

\subsection{Metodología}

La siguiente investigación trata de un estudio mixto que se elabora mediante revisiones académicas, encuestas y entrevistas.

El estudio estará compuesto tanto de análisis cuantitativo mediante encuestas, como de análisis cualitativo mediante entrevistas personales.

También contendrá un trabajo de campo que se corresponde con las entrevistas personales y las encuestas online realizadas a profesionales del sector turístico.

La metodología se completa con la información que aparece en el apartado 4.2.

\section{EVOLUCIÓN DE LOS ESTUDIOS UNIVERSITARIOS EN TURISMO}

\section{1. Ámbito español}

Realizando una breve reseña histórica, cabe destacar que no fue hasta los años sesenta cuando España empezó a percatarse de cómo la actividad turística podría convertirse en una herramienta fundamental para equilibrar la balanza de pagos, para financiar el déficit público considerando el turismo internacional o para crear empleo nacional.

Con la entrada en la Comunidad Económica Europea (CEE) en los años ochenta, se alcanzó una economía abierta e integrada. No obstante, se agravó aún más el problema estructural del déficit con este cambio tan brusco, siendo la actividad turística la que actuó como salvador del equilibrio económico español.

Esta situación es la que revalorizó el turismo, siendo desde entonces cuando se le comenzó a dar valor como actividad económica, aunque, como Gómez (Gómez, 1994) afirma, no se le dio el reconocimiento que debiese. Es esta la razón de que el Plan Marco de Competitividad del 1992-962 incluya dentro del plan de excelencia turística un objetivo de sensibilización en España sobre la importancia socioeconómica de la actividad turística.

Todo esto desencadena en una preocupación por la preparación y formación de la mano de obra que se dedicará al sector turístico. Sin embargo, no fue hasta 1957 cuando se fundó la primera escuela de turismo ubicada en Madrid por parte de una entidad docente de carácter privado, el Centro Español de Nuevas Profesiones (CENP).

Desde entonces han existido diversos títulos en turismo en España, como puede verse en la siguiente tabla.

\begin{tabular}{|c|c|c|c|c|}
\hline & TET & TEAT/DEAT & DIPLOMATURA & GRADO \\
\hline NOMBRE & $\begin{array}{l}\text { Técnico de } \\
\text { Empresas } \\
\text { Turísticas }\end{array}$ & $\begin{array}{l}\text { Técnico en Empresas } \\
\text { y Actividades } \\
\text { Turísticas/ } \\
\text { Diplomatura en } \\
\text { Empresas y } \\
\text { Actividades Turisticas }\end{array}$ & $\begin{array}{l}\text { Diplomatura en } \\
\text { Turismo }\end{array}$ & $\begin{array}{l}\text { Grado en } \\
\text { Turismo }\end{array}$ \\
\hline VIGENCIA & $1963-1980$ & $1980-2001$ & $1996-2007$ & $\begin{array}{c}2007 \text { - } \\
\text { Actualidad }\end{array}$ \\
\hline DECRETO & $\begin{array}{c}\text { Real Decreto } \\
2427 / 1963\end{array}$ & $\begin{array}{c}\text { Real Decreto } \\
865 / 1980\end{array}$ & $\begin{array}{c}\text { Real Decreto } \\
604 / 1996\end{array}$ & $\begin{array}{c}\begin{array}{c}\text { Real } \\
\text { Decreto } \\
1393 / 2007\end{array} \\
\end{array}$ \\
\hline $\begin{array}{c}\text { ÁMBITO } \\
\text { GEOGRÁFICO }\end{array}$ & $\begin{array}{l}\text { Madrid, más } \\
\text { tarde } \\
\text { Barcelona y } \\
\text { Sevilla }\end{array}$ & $\begin{array}{l}\text { Madrid, más tarde } \\
\text { Cataluña, Baleares, } \\
\text { Canarias y Andalucia }\end{array}$ & - & - \\
\hline
\end{tabular}

Tabla 1: Titulaciones estudios de Turismo en España

Fuente: Elaboración propia a partir del Libro Blanco de Turismo ANECA (2004) 
En primer lugar, en los años sesenta con el Decreto 2427/1963, del 7 de septiembre, se crea la Escuela Oficial de Turismo en la cual se regula la concesión del título como «legalmente reconocido por el Ministerio de Información y Turismo» ${ }^{2}$ a todos los Centros de enseñanza turística no oficial, esto hace que se imparta en España el título de "Técnico de Empresas Turísticas" (TET) que aparecería en ese mismo año 1963.

En segundo lugar, en 1980, los propios empresarios del sector demandaban un titulado con mayor formación y conocimientos en el ámbito empresarial, esto hizo que este mismo año apareciese, tras el Real Decreto 865/1980, del 14 de abril, el nuevo título de «Técnico en Empresas y Actividades Turísticas» (TEAT); a pesar de estar compuesto por tres años de formación como el anterior título TET, el TEAT otorgaba menos créditos, llegándose a plantear añadir un cuarto año, algo que jamás sucedió.

En 1983, una ley orgánica de reforma universitaria (11/1983) aprobará los títulos de carácter oficial que serán validos en todo el territorio español. Con respecto a este año, Torres (Torres,1993) consideraba que la formación hasta el momento se caracterizaba por ser insuficiente, incompleta, no integrada y obsoleta.

Por otro lado, ya en el 1996, en busca de esa constante mejora en la formación turístico aparecerá el Real Decreto 259/1996. Esta fecha será clave para la formación en turismo en España, puesto que marcará el inicio del título "Diplomado en Empresas y Actividades Turísticas" (DEAT), que mostraba la necesidad de mejora de muchos aspectos en la formación impartida hasta el momento, y, además, buscaba preparar y capacitar de una manera superior a los egresados, para unos directivos de las empresas turísticas del país cada vez más exigentes.

Posteriormente, se aprobó el Real Decreto 604/1996, que establecía un nuevo título oficial de «Diplomado en Turismo", con el mismo valor académico y profesional que el DEAT. Fecha muy tardía teniendo en cuenta la importancia del turismo como actividad propulsora de la economía española y siendo España uno de los principales destinos turísticos en la época.

Algunos docentes consideraban que ni siquiera la diplomatura era suficientemente satisfactoria, debido a la reducción de horas dedicadas al estudio de idiomas. Además, sumando otros aspectos, los empresarios no creían en la capacidad de la universidad para atender las necesidades que se les planteaba desde las empresas.

Por último, el grado aparecerá después del afamado "Proceso Bolonia"3 y el proceso de Espacio Europeo de Educación Superior (EEES) llevado a cabo desde 1999.

El Grado en Turismo queda regulado en el Real Decreto 1393/2007, este deberá completarse con un postgrado o Máster y más adelante podrá completarse la formación con un doctorado.

\subsection{El caso de la universidad de Sevilla: de la diplomatura en turismo al grado en turismo}

Si en España la formación en turismo fue tardía, aún más lo fue para el caso de Sevilla, no siendo hasta el 1961 cuando el Centro Español de Nuevas Profesiones (CENP) inaugura la tercera Escuela de Turismo de España en Sevilla.

Volviendo al tema que concierne a esta investigación, el 2 de septiembre de 1999, se lleva a cabo una resolución por parte de la Universidad de Sevilla por la que se ordena una publicación de un plan de estudios conducente a la obtención del título de Diplomado en Turismo, lo que hoy en día recordamos como "La Diplomatura en turismo" y que se impartiría en la Escuela Universitaria de Estudios Empresariales de Sevilla.

Esto queda estipulado en el Real Decreto 604/1996 de 15 de abril; En el mismo se menciona expresamente que «las enseñanzas conducentes a la obtención del título de Diplomado en Turismo deberán proporcionar la formación teórico-práctica adecuada para la gestión de organizaciones e instituciones relacionadas con el turismo». Según el Real Decreto español, a partir de las bases que quedan estipuladas en este decreto el plan de estudios de Sevilla debería amoldarse a este.

En la Universidad de Sevilla se esforzaban por inculcar competencias específicas de carácter económico, todas ellas casi idénticas a las competencias que ya se enseñaban en la escuela empresarial, solo que esta vez encaminadas a las empresas turísticas.

Observando el plan de estudios de la diplomatura en turismo, queda claro el peso que suponen las materias relacionadas con la gestión y dirección de empresas, muy ligado a las raíces de la escuela de Sevilla que siempre había sido una escuela empresarial.

\footnotetext{
2 “El Ministerio de Información y Turismo" se creó por Decreto-Ley de 19 de julio de 1951, por el que se reorganizó la Administración Central del Estado.

3 Proceso Bolonia; Se trata de un proyecto impulsado por la Unión Europea con el objetivo de armonizar los sistemas universitarios de los países miembros y eliminar los complejos procedimientos para la homologación de títulos.
} 
El Grado en Turismo aparece junto al nuevo Plan Bolonia, pero en este caso al igual que con las otras titulaciones en la ciudad sevillana el grado se implantaría en el 2010, habiendo quedado regulado en España desde el 2007.

A diferencia de la diplomatura, el Grado incrementará materias relacionadas con el arte y las humanidades, entre otras muchas competencias que tendrían que justificar un año más de formación con respecto a la anterior diplomatura en turismo, siendo las letras las que ocuparán esa formación "de relleno" que completará el nuevo grado de turismo.

A primera vista parece ser una gran mejora para la universidad de Sevilla, puesto que estos estudios se han visto incrementados un año más, mejorando la formación de los estudiantes de turismo, pero la realidad parece ser otra, debido a que el grado en turismo de la Universidad de Sevilla ha perdido su verdadera esencia empresarial, provocando una gran intrusión por parte de los estudiantes de Administración y Dirección de Empresas en el ámbito laboral de los estudiantes de turismo, que ahora con el grado pierden esa especialización en el área empresarial, desencadenando todo esto en que, esta vez, sean los egresados de turismo los que provoquen intrusión en los empleos que van encaminados a los estudiantes de formación profesional del sector turístico.

\section{EMPLEABILIDAD DEL SECTOR}

\subsection{Empleabilidad: concepto}

Yorke (2004)

"La empleabilidad está compuesta por un conjunto de logros-habilidades, conocimientos y atributos personales que aumentan la probabilidad de los graduados de encontrar un empleo y de tener éxito en sus puestos, lo cual les beneficia a ellos mismos, a la fuerza de trabajo, a la comunidad y a la economía"

Como bien se puede ver en la definición citada al comienzo de esta sección, la empleabilidad implica una serie de habilidades, aptitudes y conocimientos entre otros aspectos que hacen que el graduado tenga más posibilidades.

\subsection{Mapa de empleabilidad}

Es una gran cantidad de empleo el que se genera en el sector turístico dentro del estado español, pero el tipo de empleo que se genera no es de los más estables en España. La Encuesta de Inserción laboral de Titulados Universitarios realizada por el INE nos arroja la siguiente información:

"Los graduados en Turismo registraron los mayores porcentajes de contratos temporales (70,8\%)"

El estudio sobre el empleo en el sector turístico español llevado a cabo por Exceltur confirma este aspecto añadiendo que el porcentaje de trabajadores temporales en bares, restaurantes y cafeterías suponen un 39,6\%, por lo que respecta al alojamiento un $37,4 \%$ y en el transporte de viajeros se sitúa en un $20,1 \%$.

Todo esto a pesar de que un informe oficial de Coyuntura del 2014, realizado por el ministerio de Industria, Energía y Turismo afirmaba un incremento y tendencia positiva basándose en los datos de afiliación de la Seguridad Social.

Gonzalo Fuentes, dirigente de $\mathrm{CCOO}$, afirma que, aunque sí se genera empleo, este es de poca calidad, precario y a tiempo parcial.

Pero algo que interesa mucho más, independientemente del tipo de empleo que se genere en nuestro sector, es que en España hay muchísimas personas con estudios superiores trabajando en puestos de baja cualificación, perjudicando gravemente a los egresados españoles en general, según un informe de la Fundación Conocimiento y Desarrollo del 2016 (CYD). 


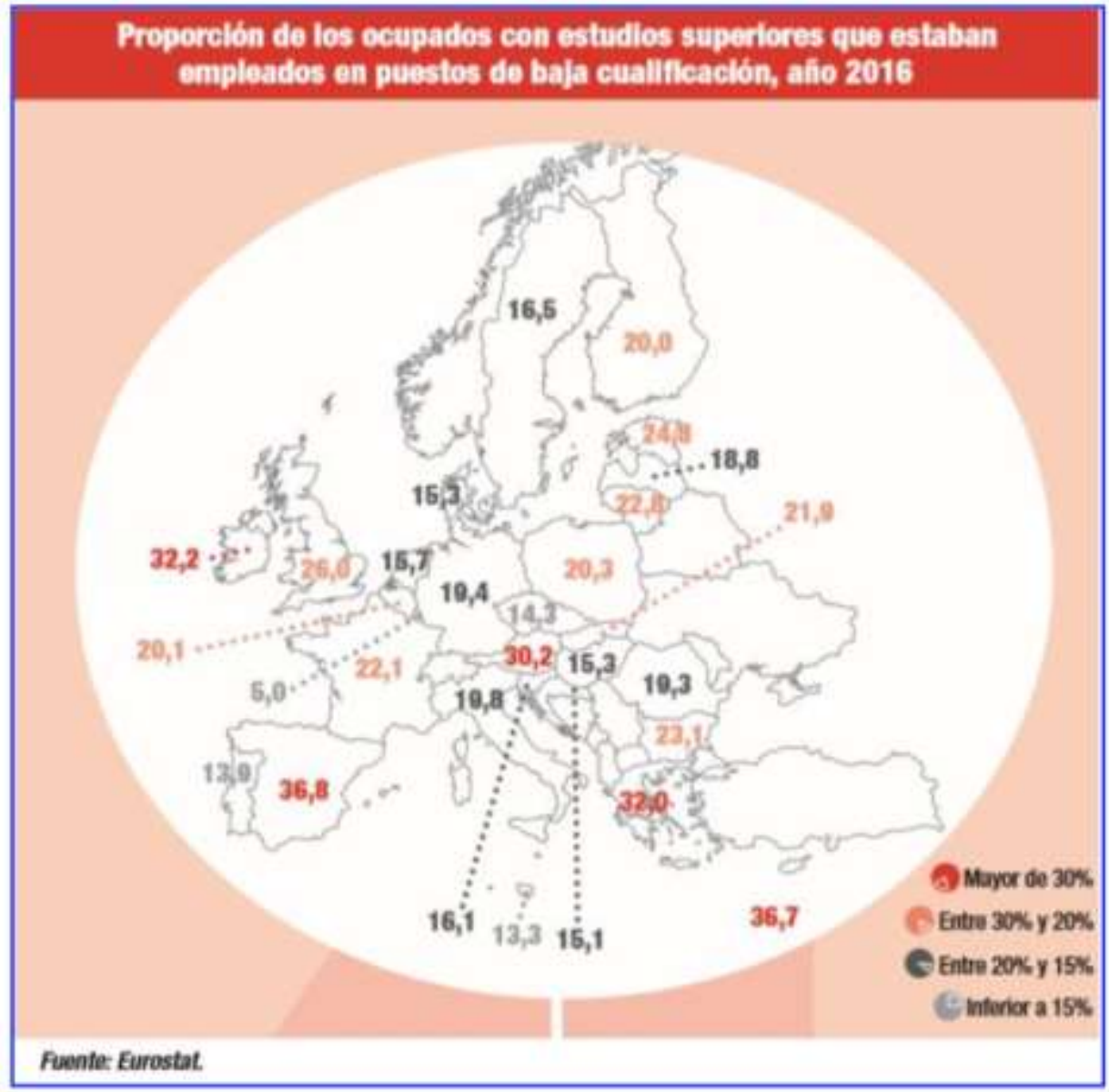

Imagen 1: Ocupados con sobre cualificación Europa

Fuente: CYD (2016)

Lo peor que puede observarse con relación a esto, es que una de las titulaciones que tienen más dificultad para encontrar trabajo acorde con su nivel formativo es la de Turismo, según el primer informe de inserción laboral de los egresados universitarios, realizado por el Ministerio de Educación, cultura y deporte.

En el próximo gráfico, obtenido del Libro Blanco del Grado en turismo (ANECA, 2004:47), se especifican las cualificaciones de los profesionales según el nivel de estudios. Como puede observarse, el grado de turismo debería aportar conocimientos y preparación más que suficientes como para cubrir puestos de características como: Cargos intermedios y Dirección de operaciones en cualquier empresa.

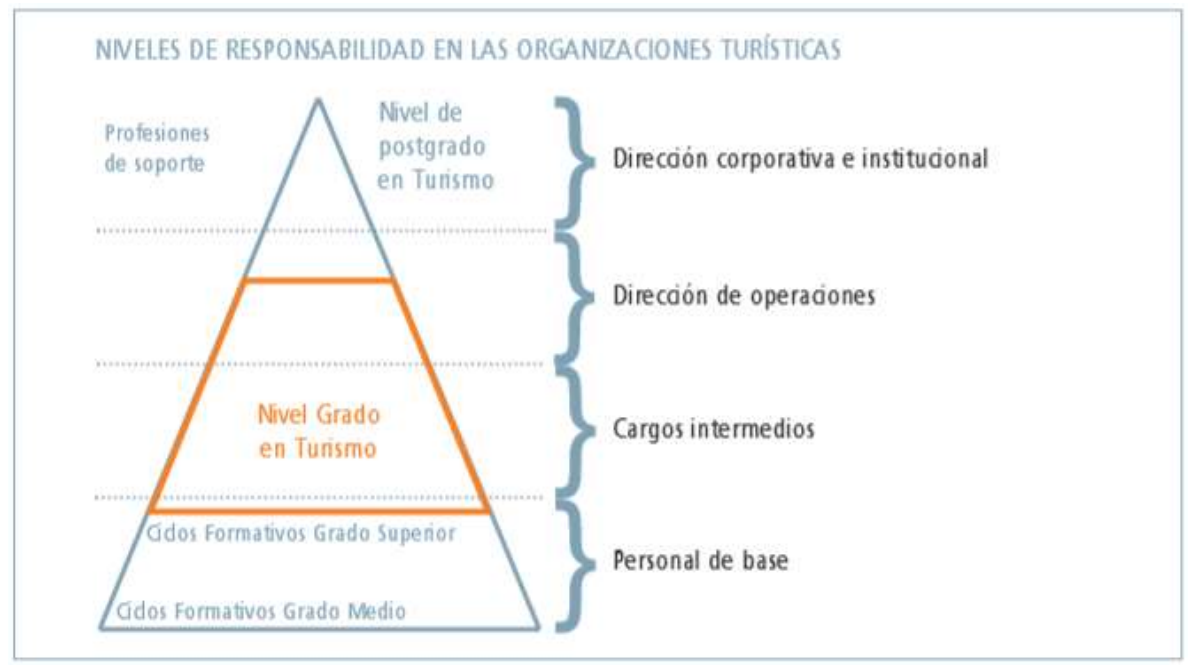

Imagen 2: Niveles responsabilidad organizaciones turísticas Fuente: Libro Blanco del Turismo ANECA (2004) 
Sin embargo, los datos actuales muestran que la realidad es muy diferente a lo esperado; actualmente muchos egresados en turismo terminan ocupando puestos de personal de base, los cuales están encaminados a estudiantes de ciclos formativos tales como: Grado Superior y Grado Medio.

También la amplia necesidad de personal para tareas de menor cualificación (ya sean limpiadoras, camareras de piso...) explica por qué de esta sobre cualificación dentro del sector turístico.

Sin embargo, cabe cuestionarse la siguiente pregunta, ¿Es este el principal factor de la sobre cualificación o afecta en cierta medida el posible hecho de que los graduados en turismo no sean los que ocupan los puestos destinados para ellos?

\subsection{Formación universitaria dual como instrumento de inserción laboral: ejemplo Alemania y Francia.}

Esto nos lleva a querer conocer qué herramientas utilizan otros países para que los empleados con estudios superiores no terminen en puestos de baja cualificación en niveles tan altos como ocurre en el caso español, además de ayudar a la inserción de jóvenes egresados.

Universidades alemanas utilizan un sistema que ellos llaman: The German Vocational Training System, también conocido como formación universitaria dual, en el cual se integran los procesos de enseñanza y aprendizaje tanto en la universidad como en una empresa siendo esto una alternancia entre las horas lectivas y las horas prácticas en una empresa durante toda la etapa universitaria.

El origen de este tipo de formación dual en educación superior se sitúa en la década de los setenta con la creación de la Berufsakademie (Universidad Empresarial) en Baden-Württember (Alemania).

Incluso tenemos constancia de algunos datos que nos muestran el porcentaje de alumnos alemanes egresados que una vez finalizados sus estudios de formación dual son contratados por aquellas empresas en las que estaban trabajando; en el informe del CYD se afirma que;

"En los últimos dos años, más del 85\% de los graduados han conseguido un contrato de trabajo indefinido con la graduación (2014-2016)"

Y es que en estos países está siendo muy efectivo este tipo de formación en la cual la vida de un estudiante está muy ligada a la empresa a la que se dedica desde el primer año de su formación, fomentando que el estudiante crezca en la misma, que aprenda con ella y aplique sus conocimientos teóricos a la práctica durante todo su aprendizaje; todo esto suele desencadenar en que este, una vez finalizada su formación termine por ser contratado por la empresa en la que ha permanecido ese periodo.

\section{ANÁLISIS EMPÍRICO: ¿QUÉ VALORAN LAS EMPRESAS EN LOS EGRESADOS UNIVERSITARIOS EN TURISMO?}

\section{1. ¿Qué valoran las empresas en los egresados universitarios españoles? Estudios previos}

El cuatro de abril de 2018, Hosteltur publicó un artículo en el que se presentan los resultados de un informe realizado por Turijobs a más de 200 empresas del sector turístico para saber cuáles son los factores que más valoran las empresas a la hora de contratar estudiantes recién graduados en hostelería y turismo.

los idiomas son lo que las empresas turísticas más valoran, convirtiéndose en un requisito primordial. Entre ellos se destaca inglés, alemán y francés entre los más demandados.

Las competencias transversales tales como: Trabajo en equipo, resolución de conflictos, toma de decisiones o adaptación a nuevos proyectos son aspectos, que, aunque no se encuentren en primera posición de los requisitos de las empresas, no dejan de suponer un $38,46 \%$ de los resultados del informe llevado a cabo por Turijobs.

Un tercer aspecto muy valorado por las empresas del sector, es que estos estudiantes hayan realizado un erasmus o tengan experiencia laboral en el extranjero; los empresarios afirman que esta experiencia que proporciona la estancia en otros países y la visión global que adoptan se convierte en un factor muy valorado para ellos.

Pero a pesar de todos los resultados, Xavier Martín (director de Turijobs) afirma que;

"Las empresas del sector valoran la actitud por encima de todas las capacidades. Con esta, los estudiantes pueden llegar a donde quieran"

Por otro lado, el cuatro de mayo de 2018, hosteltur publica un artículo en el que se habla de las actuales necesidades de las empresas turísticas; tanto Carlos Díez de la Lastra, director general de la Escuela Les Roches Marbella, Fernando de Pablo, presidente de Segittur como Arturo Bernal, gerente de Turismo Costa del Sol, formaron parte de una mesa redonda centrada en "Turismo y Big Data". 
Carlos Díez de la Lastra, afirma que la tecnología de datos hoy día será lo que marque el futuro de nuestra industria, que nos encontramos ante las autopistas de la tecnología y debido a que en la actualidad tenemos acceso a enormes cantidades de información que es necesario filtrar, las empresas buscan talento capaz de gestionar la transformación digital que está viviendo el sector.

El Big Data forma parte del presente y es más que necesario prepararse para su gestión si se quiere seguir siendo una potencia turística mundial.

Carlos Díez incide en que es necesario un consenso entre los actores de la industria para impulsar la formación de los profesionales del turismo de modo que se eleve la calidad del servicio en todos los tramos de la cadena, destacando que aquellos hoteles que más invierten en formación serán los que obtengan mejores resultados.

También añadió que;

"A pesar del esfuerzo de pymes y pequeños hoteles por unirse a esta transformación, sólo aquellos capaces de invertir en formación específica podrán competir con las plataformas surgidas en el entorno online y adelantarse a las necesidades del turista del futuro"

Carlos Díez de la Lastra.

Cada vez entran más en juego competencias transversales relacionadas más con el comportamiento y la forma de ser de la persona que con el conocimiento teórico en el sentido estricto de la palabra; así como nuevas competencias relacionadas con las tecnologías más actuales, que incitan a una revolución educativa en la que se dote a los estudiantes de las capacidades necesarias que permitan su empleabilidad en un nuevo y prometedor mercado emergente.

\section{2. ¿Qué valoran las empresas en los egresados en turismo de la universidad de Sevilla?}

En este apartado se van a mostrar los resultados empíricos obtenidos tras la realización de encuestas y entrevistas a profesionales del sector turístico.

Para clarificar la forma de proceder se muestra a continuación la siguiente tabla en la que se recoge la estructura seguida en este análisis empírico.

\begin{tabular}{|c|c|c|}
\hline \multicolumn{3}{|c|}{ MÉTODOLOGIAA EMPLEADA } \\
\hline \multirow{2}{*}{$\begin{array}{l}\text { OBTENCION DE } \\
\text { INFORMACION }\end{array}$} & \multicolumn{2}{|l|}{ Encuestas (*) } \\
\hline & \multicolumn{2}{|c|}{ Entrevistas personales (") } \\
\hline \multirow[t]{2}{*}{ MUESTREO } & Muestra & $\begin{array}{l}\text { Empresarios/profesionales } \\
\text { del sector turistico que } \\
\text { acogen estudiantes en } \\
\text { prácticas del Grado en } \\
\text { Turismo de la US }\end{array}$ \\
\hline & Tamaño Muestra & $\begin{array}{l}16 \text { encuestas } \\
4 \text { entrevistas personales }\end{array}$ \\
\hline \multirow[b]{2}{*}{ TRABAJO DE CAMPO } & Periodo & mayo 2018 - julio 2018 \\
\hline & Lugar & $\begin{array}{l}\text { Sede de las empresas y } \\
\text { mediante correo } \\
\text { electrónico }\end{array}$ \\
\hline \multirow{2}{*}{$\begin{array}{l}\text { ANĀLISIS DE LA } \\
\text { INFORMACIÓN }\end{array}$} & Técnicas estadísticas & Descriptivas \\
\hline & Programa informático & EXCEL (2016) \\
\hline
\end{tabular}

Tabla 2: Metodología

Fuente: Elaboración propia

$\left(^{*}\right)$ Las encuestas y las entrevistas se incorporan como anexos a este trabajo.

\subsection{1. ¿Qué valoran las empresas? Resultado de las encuestas.}

Los cuestionarios fueron lanzados a 55 de los responsables de personal o recursos humanos regionales de las empresas que tienen convenio con la Universidad de Sevilla para la realización de las prácticas de los alumnos del grado en turismo; pero se debe añadir que ha habido una gran dificultad para obtener respuesta por parte de estos empleadores, de los cuales 16 han respondido finalmente a las preguntas propuestas en la encuesta.

Esto representa un $29,09 \%$ de tasa de respuesta, aunque es importante recalcar que, en estas 16 empresas, que suponen ese porcentaje del total, realizan sus prácticas ni más ni menos que 129 alumnos del grado en turismo. 
Incluso nos encontramos con que algunas de estas empresas tienen al año unos 60 alumnos en prácticas, quedando claro el peso que suponen las declaraciones de estos profesionales.

En cuanto al nivel de satisfacción con el que se encuentran estos responsables una vez realizadas las prácticas por el alumno, los resultados obtenidos fueron: para muy satisfecho siendo su valor 5 el porcentaje es de un $18,8 \%$ (3 empleadores), para bastante satisfecho con un valor de 4 el porcentaje es de un $50 \%$ ( 8 empleadores) y para satisfecho con un valor de 3 , el porcentaje es de un $31,3 \%$ (5 empleadores).

Una de las preguntas que desvelan más información, es aquella relacionada a las competencias transversales y las específicas; por lo que respecta a las primeras, se les enumera a los encuestados unas ciertas competencias transversales tales como: Capacidad de organización y planificación, conocimiento de una lengua extranjera, conocimientos de informática relativa al ámbito de estudio, resolución de problemas y toma de decisiones y trabajo en equipo entre otras; algunas de las respuestas son las siguientes:

\begin{tabular}{|l|c|}
\hline \multicolumn{1}{|c|}{ COMPETENCIAS } & PORCENTAJE (\%) \\
\hline $\begin{array}{l}\text { Capacidad de organización y } \\
\text { planificación }\end{array}$ & $37,5 \%$ \\
\hline Conocimiento de una lengua extranjera & $87,5 \%$ \\
\hline $\begin{array}{l}\text { Conocimientos de informática relativa } \\
\text { al ámbito de estudio }\end{array}$ & $0 \%$ \\
\hline Resolución de problemas & $81,3 \%$ \\
\hline Toma de decisiones & $50 \%$ \\
\hline $\begin{array}{l}\text { Trabajo en equipo } \\
\text { Habilidades en las relaciones } \\
\text { interpersonales }\end{array}$ & $18,8 \%$ \\
\hline Razonamiento critico & $31,3 \%$ \\
\hline Compromiso ético & $18,8 \%$ \\
\hline Adaptación a nuevas situaciones & $6,3 \%$ \\
\hline Creatividad & $18,8 \%$ \\
\hline Liderazgo & $6,3 \%$ \\
\hline Iniciativa y espiritu emprendedor & $12,5 \%$ \\
\hline
\end{tabular}

Tabla 3: Competencias transversales

Fuente: Elaboración propia (2018)

Estos porcentajes indican aquellos encuestados que consideran que estas competencias nombradas necesitan ser mejoradas.

Así, el conocimiento de una lengua extranjera es la que según los encuestados debe ser mayormente mejorada contando con un aplastante $87,5 \%$ y también la resolución de problemas cuenta con un número elevado como lo es un $81,3 \%$; siendo los conocimientos informáticos con un $0 \%$, y el compromiso ético y la creatividad con un $6,3 \%$ los que consideran que necesitan menor mejora.

Con respecto a las competencias específicas, los resultados fueron los siguientes (donde nuevamente se indica el porcentaje de profesionales que opinan que dicha competencia necesita ser mejorada): 


\begin{tabular}{|c|c|}
\hline COMPETENCIAS & PORCENTAJE $(\%)$ \\
\hline Manejo fluido lengua inglesa & $81,3 \%$ \\
\hline $\begin{array}{c}\text { Comunicación de manera oral y escrita } \\
\text { de segunda lengua extranjera }\end{array}$ & $75 \%$ \\
\hline $\begin{array}{l}\text { Conocimiento y aplicación de } \\
\text { conceptos básicos en materia de: } \\
\text { Derecho, Economia, Geografia, } \\
\text { Estadistica y Dirección de empresas, } \\
\text { entre otros. }\end{array}$ & $0 \%$ \\
\hline $\begin{array}{l}\text { Análisis dimensión e impactos } \\
\text { económicos generados por el turismo }\end{array}$ & $0 \%$ \\
\hline $\begin{array}{c}\text { Management, dirección y gestión de } \\
\text { los distintos tipos de entidades } \\
\text { turísticas }\end{array}$ & $25 \%$ \\
\hline $\begin{array}{l}\text { Comprensión del marco legal que } \\
\text { regula las actividades turísticas }\end{array}$ & $6,3 \%$ \\
\hline $\begin{array}{l}\text { Conocimiento y aplicación de las } \\
\text { tecnologias de la información y las } \\
\text { comunicaciones }\end{array}$ & $12,5 \%$ \\
\hline $\begin{array}{l}\text { Comprensión de procesos de } \\
\text { innovación y desarrollo de la empresa } \\
\text { y fomento del espiritu emprendedor. }\end{array}$ & $12,5 \%$ \\
\hline $\begin{array}{l}\text { Capacidad de aplicación } \\
\text { conocimientos teóricos, } \\
\text { metodológicos y técnicos a la práctica. }\end{array}$ & $31,3 \%$ \\
\hline $\begin{array}{c}\text { Orientación de las relaciones sociales } \\
\text { en la organización }\end{array}$ & $18,8 \%$ \\
\hline
\end{tabular}

Tabla 4: Competencias específicas

Fuente: Elaboración propia (2018)

De este modo, se observa como el manejo de la lengua inglesa abarca un 81,3\%, la comunicación oral y escrita de una lengua extranjera se posiciona con un $75 \%$ y la capacidad de aplicar los conocimientos teóricos, metodológicos y técnicos a la práctica implican un 31,3\%, como datos más significativos.

En base a las competencias transversales, 13 de los empresarios que han respondido la encuesta afirman que estas deben ser mejoradas en mayor medida mientras que 3 de los empresarios afirman que deben ser mejoradas en menor medida.

$Y$ en base a las competencias específicas, 12 afirman la necesidad de mayor medida de mejora, 3 responden que la mejora debe ser en menor medida y se encuentra un resultado de 1 encuestado que afirma no ser necesaria ninguna mejora en este tipo de competencias,

Teniendo toda esta información se procedía en la encuesta a preguntar cual es definitivamente la precepción de los empleadores con respecto a la productividad de los alumnos en prácticas del grado en turismo, siendo 5 profesionales los que responden que la productividad percibida es alta, implicando un total de un 31,3\%; 9 de los profesionales consideran que la productividad de los alumnos es media, suponiendo un $56,3 \%$ y por último, 2 respuestas opinan que la productividad es baja, con un 12,5\%; pero ninguna respuesta que refleje una productividad nula.

La penúltima cuestión dejaba una pregunta abierta en las que los profesionales de recursos humanos deberían especificar cuál creen que podría ser el "Talón de Aquiles" de estos alumnos. Muchos de ellos afirman que el idioma es una de las limitaciones más grandes, otros confiesan que hay un miedo escénico al trato directo con el cliente por parte del alumno y poca iniciativa, muchos comentan una actitud negativa por parte de alumnos españoles, pero no por parte de los alumnos en prácticas extranjeros, falta de compromiso con las prácticas debido a no ser remuneradas, falta de responsabilidad, etc.

Y la última cuestión, va dirigida a averiguar qué deben poseer estos alumnos para ser considerados como posibles empleados de estas empresas encuestadas; ante todo se recalcan aspectos relacionados con la actitud, tales como: sentido de la responsabilidad, madurez, buena voluntad, compromiso; también algunas competencias como: El idioma, resolución de conflictos, trabajo en equipo, capacidad de organización; y algunos 
aspectos más como: aceptación de las circunstancias laborales del sector, aceptación de las críticas... entre otros.

\subsection{2. ¿Qué valoran las empresas? resultado de las entrevistas}

Las entrevistas realizadas a cuatro personas especializadas en el sector turístico todavía desvelan más información que las encuestas; en total estos cuatro empleadores cuentan con 60 alumnos en prácticas anualmente en su empresa, siendo Meliá el que más con un número de 40 alumnos, le sigue Barceló con 10 alumnos, CONTURSA FIBES con 6 alumnos y la agencia de viajes B The Travel Brand con 4 alumnos anualmente.

Aquellos que tienen más experiencia en el sector y en su puesto son Elena Moros Diaz y Fernando Bocardo Crespo, con 30 y 28 años de experiencia respectivamente, siendo su opinión muy importante puesto que conocen sobradamente el sector al que se dedican, habiendo afirmado ambos dedicarse, además, por vocación. Por lo que respecta a Enrique Castilla Gavilán, es un profesional joven con muchas ganas y capaz de identificar con facilidad el talento de las personas, con 4 años en su puesto afirma dedicarse a lo que realmente le apasiona.

Ante la primera pregunta que se les plantea, si quedan o no satisfechos con los alumnos en prácticas, todos coinciden en que en términos generales sí quedan satisfechos, aunque bien es cierto que hay matices. Muchos de nuestros profesionales comentan que en algunas ocasiones no quedan satisfechos al $100 \%$, pero son pocas las veces en las que esto acontece; muy relacionada a esta cuestión, se pregunta por la actitud de estos alumnos en las prácticas a lo que responden que la actitud suele ser positiva, pero hay algunos alumnos que consideran las prácticas como algo más que forma parte de la carrera universitaria y no se lo toman demasiado enserio.

A partir de la siguiente pregunta se pretende conseguir dar respuesta en mayor medida a los objetivos de esta investigación, y es aquí donde se intenta averiguar cuáles podrían ser las mejoras con respecto a competencias específicas y las competencias transversales en las prácticas, de modo que estos alumnos sí sean tenidos en cuenta para su contratación en la empresa; Elena Moros y Carmen Alonso coinciden en que por lo que respecta a competencias específicas es muy normal que los alumnos en principio lleguen algo perdidos, en general afirman que estas competencias específicas deben ser adquiridas con la puesta en práctica de las labores diarias de la empresa puesto que hay programas y un know-how muy específico dentro de sus compañías, con ejemplos como el programa de reservas OPERA, o el que utilizan en la agencia de viajes de B The Travel Brand.

En cambio, Fernando Bocardo y Enrique Castilla, a pesar de coincidir en algunos aspectos con Elena y Carmen, consideran que hay una tara muy grande en los alumnos en prácticas y es el idioma, recalcan que no son excesivamente estrictos pero que sí pedirían que estos alumnos tuviesen mínimo un B2 de inglés, ya que las características del sector y el tipo de empresas al que se dedican así lo requiere.

En relación con las competencias transversales, muchos de nuestros entrevistados dicen que los alumnos llegan un poco retraídos, esperan a recibir indicaciones, no son muy proactivos, ni mucho menos resolutivos; aunque Elena y Enrique aseguran que estas competencias son inherentes a la persona y que en un mes puede verse quién tiene talento, facilidad para adaptarse, compromiso con la empresa y quién por el contrario no. Dada su importancia, es en esta respuesta en la que los entrevistados se basan para responder a la pregunta sobre qué debe tener este alumno para ser tenido en cuenta por su empresa.

Elena Moros Díaz asegura que ellos pedirían mínimo estos aspectos: Empatía con el cliente, proactividad y capacidad resolutiva por parte del alumno; Fernando Bocardo en cambio opina que sería necesaria la capacidad de rápido aprendizaje y mejora importante del idioma (inglés), Enrique Castilla incide en que Meliá valora ante todo el esfuerzo, el compromiso y la ilusión.

Una vez cumplen estos requisitos la pregunta siguiente está relacionada con la posición jerárquica a las que estos alumnos contratados pueden optar en la empresa teniendo, nada más, el grado de turismo; Elena Moros comenta que en Barceló apuestan por la búsqueda del talento, con lo cual, aunque los alumnos comiencen en puestos de ayudante de recepción o similares, con experiencia y tiempo pueden acceder a puestos de dirección. Enrique Castilla añade que muchos de los jefes de Meliá Lebreros son diplomados en turismo, que en Meliá los alumnos comienzan cubriendo vacaciones en verano y que si estos demuestran valer se les va prorrogando el contrato, anualmente van valorando las capacidades de estos alumnos que ya han sido contratados y se les plantea un plan de carrera en la propia empresa, aunque incide que avanzar en Meliá es avanzar en la compañía, no solo en el Meliá Lebreros.

Ante la cuestión sobre que piensan estos profesionales con respecto al $76,5 \%$ de sobre educación de los egresados en turismo que el informe de la Fundación Conocimiento y Desarrollo del 2016 nos muestra, todos los profesionales están más que de acuerdo en que el alumno comienza desde abajo y en el sector turístico mínimo se exige el graduado en turismo para ejercer como ayudante de recepción, esto no quita que estos alumnos tengan la oportunidad de ascender a mejores puesto en la empresa acordes a su formación, siempre que estos 
alumnos contratados tengan aquello que la empresa busca. Todos reconocen que en la mayoría de los casos son más las variables endógenas las que afectan a la contratación de los egresados que las exógenas; inciden en que puede haber crisis o no, pero trabajo hay y quien vale trabaja.

La formación dual ha sido un tema interesante que alguno de los entrevistados desconocía, afirman que suena muy bien y que ellos como empresa si acogerían a estos alumnos para que estuviesen con ellos durante esos cuatro años de formación, aunque como Elena Moros comenta ni siquiera la formación dual facilita que el alumno conozca la realidad del sector turístico porque seguiría trabajando de lunes a viernes, cuatro horas y en el mismo turno; Fernando Bocardo piensa que este sistema dual favorecería mucho la contratación de estos alumnos siempre y cuando sea un número inferior al número de alumnos que tienen anualmente de prácticas en la empresa, si no esto sería imposible.

También hay una pregunta encaminada a averiguar si fuese necesaria una mayor colaboración entre las empresas del sector y la Universidad de Sevilla, y ante esta pregunta la respuesta es unánime, sí debería haber mayor colaboración y sobre todo la Universidad debería de interesarse por averiguar cuáles son las verdaderas necesidades de las empresas y esto desencadena en la última cuestión de la entrevista, que deja al entrevistado la posibilidad de dar su feedback al plan de estudios de la Universidad de Sevilla:

"Falta mucha información con respecto a lo que es la carrera de turismo. ¡Turismo es trabajar duro! Mientras otros se divierten... tal vez se deba recalcar esto, para poder obtener buenos profesionales, que se dediquen a este sector por verdadera vocación"

Elena Moros, responsable regional de recursos humanos de hotel Barceló.

"Se imparten muchas materias con un contenido excesivamente teórico que seguramente a los pocos meses ya lo has olvidado y poca materia práctica"

Fernando Bocardo, jefe de personal CONTURSA FIBES.

“El tiempo de las prácticas es escaso, los turnos limitados... la empresa no llega a conocerse por el alumno, ni el puesto, ni el sector. No es lo mismo un turno de mañana que de tarde que de noche, ni siquiera los fines de semana"

Enrique Castilla, Jefe Departamento de Recursos Humanos hotel Meliá Lebreros.

\section{CONCLUSIONES Y RECOMENDACIONES FINALES}

\subsection{Conclusiones}

Para concluir este estudio, en este último capítulo se van a resumir los puntos clave obtenidos en el trabajo de campo.

Desde el inicio pretendíamos averiguar cuáles eran las variables que podían afectar a la contratación de los egresados en turismo, llegando a la conclusión que más que factores externos son internos, pero no precisamente es la educación impartida en el Grado de Turismo la que plantea el mayor problema.

Bien es cierto que el plan de estudio debería modificarse en algunos aspectos, teniendo en cuenta que muchas de las recomendaciones de los profesionales entrevistados desembocan en una mejora en el uso de una lengua extranjera, en particular el inglés.

"No es cuestión de hablar tres idiomas, no exigimos eso, pero si un buen nivel de inglés" Fernando Bocardo, jefe de personal CONTURSA FIBES.

El grado permite terminar con un nivel B1 en inglés, pero todos los entrevistados coinciden en que mínimo los egresados en turismo deberían poseer un nivel B2 en esta lengua.

También hay que añadir que en muchas ocasiones las prácticas no son del todo realistas, puesto que el sector turístico no es un sector en el cual las horas laborales queden comprendidas tan solo entre las 9:00h a 21:00h de lunes a viernes y esto hace que el alumno ya egresado nunca conozca la realidad de un empleo sacrificado como lo son aquellos integrados en el sector turístico.

"Tres meses no son suficientes para conocer un puesto de trabajo, cuando realmente empieza a conocerse, las prácticas ya están finalizando"

Enrique Castilla, Jefe Departamento de Recursos Humanos hotel Meliá Lebreros.

Como el jefe de recursos humanos del Hotel Meliá Lebreros afirma, no es simplemente el desconocimiento de la realidad del sector, sino el escaso periodo de tiempo dedicado a las prácticas de empresa que se realizan en la Universidad de Sevilla, ante esta situación se planteaba la formación dual que se imparte tanto en Alemania 
como Francia, afirmando varios de estos profesionales que si apostarían por estos alumnos que crecerían dentro de la empresa durante los cuatro años que supone el Grado de Turismo.

Un aspecto también fundamental es la necesidad de una mayor cooperación por parte de las empresas y la Universidad de Sevilla, para adaptar de algún modo a los alumnos que vayan a realizar prácticas con el puesto que se ofrece en especial dentro de la compañía, debido a que no es lo mismo un puesto en un departamento de reservas en el back-office, que un puesto de recepcionista en el que se debe estar cara al público, como tampoco es lo mismo un hotel cinco estrellas como lo pueda ser uno de dos estrellas o una pensión. De este modo los alumnos que optan a las prácticas deben satisfacer los requisitos de la propia empresa y no basarse solamente en una media académica.

Sin embargo, a pesar de algunas carencias en el plan de estudios, el factor más importante resulta ser la actitud por parte de estos egresados.

En el estudio se ha hablado de que los egresados en turismo tienen un índice del $76,5 \%$ de sobre educación, esto se debe mayoritariamente a la actitud de estos egresados. La falta de experiencia por parte de estos egresados hace que las empresas los contraten en puestos de trabajo para los que no es necesaria tanta formación para desempeñar esa labor, el problema surge en el momento en que la actitud no es la más idónea para seguir avanzando en la compañía puesto que algunos profesionales entrevistados afirman lo siguiente:

"En Barceló si damos posibilidad de ascender, ofrecemos un plan de carrera, pero esto depende de la persona; Barceló se dedica a captar talentos y eso se ve a la semana de trabajo del alumno. En esa semana somos capaces de saber si esa persona es válida o si no lo es. Puede incluso llegar a dirección"

Elena Moros, responsable regional de recursos humanos de hotel Barceló.

"Hay posibilidad de desarrollo, muchos jefes de algunos departamentos de nuestro hotel son diplomados en turismo. Esto lo vamos valorando de manera anual y la empresa da oportunidad a un plan de desarrollo en la compañía, aquí no se valora si la persona tiene un grado o un máster o un título de actividades turísticas... Valoramos como se desenvuelve en su puesto, su desempeño"

Enrique Castilla, Jefe Departamento de Recursos Humanos hotel Meliá Lebreros

Es bastante evidente que las posibilidades que dan las grandes empresas en cuanto a desarrollo profesional son amplias, pero esto va a depender en gran medida de la actitud del egresado.

En el estudio se ha pretendido profundizar mucho en el tipo de competencias que deberían ser mejoradas por el alumno, cuando en realidad llegamos a la conclusión de que se valora más la actitud del alumno mediante su desempeño que la propia formación teórica.

\subsection{Recomendaciones finales}

Una vez concluido el apartado anterior, queda clara la necesidad de implantar mejoras para tratar de resolver el problema que se plantea en este estudio, no solo a nivel educativo, sino también a nivel personal.

Por lo que respecta al ámbito académico, resulta más que necesaria una mejora en el nivel de una lengua extranjera, en especial el inglés, debido a que, como ya se ha comentado en apartados anteriores, durante el grado en turismo impartido por la Universidad de Sevilla, no es hasta el segundo año de carrera cuando se comienzan a impartir clases de idiomas.

Ante esta situación, sería interesante replantearse el comienzo del aprendizaje de otro idioma desde el primer año de carrera, además de forzar a que el nivel de los egresados se asemeje al título oficial B2, al menos en inglés, idioma fundamental en el trato con personas de todo el mundo, puesto que no debemos obviar que el sector turístico no solo implica tener que poder ponernos en contacto con clientes de todo el mundo, sino que permita también la movilidad internacional de los estudiantes del Grado de Turismo.

Además, otra posible mejora podría estar enfocada a la organización de las prácticas en empresas, dando a esta mayor importancia de la que tienen actualmente. De este modo, se propondría la posibilidad de incrementar el periodo de prácticas de tal forma que quedasen repartidas a lo largo de los cuatro años de carrera o por lo menos en los tres últimos, siguiendo como modelo la formación dual implantada en otros países, aunque no teniendo porque ser exactamente del mismo modo.

En cuanto a la actitud, la principal mejora que se propondría consistiría en informar en mayor medida acerca de las salidas profesionales y contenido del Grado de Turismo, las características del sector, el tipo de contratación, etc. Así, el futuro egresado estaría totalmente informado de lo que le deparará el mundo laboral, de manera que pueda afrontarlo con una actitud más positiva, además de fomentar que estos se dediquen al sector por verdadera vocación. 
Todo esto permitiría mejorar la actitud con la que los estudiantes afrontan las prácticas y, posteriormente, su futuro profesional. No obstante, la actitud positiva debería ser algo innato del estudiante, ese algo que le aporte la fuerza necesaria para incrementar sus conocimientos, pero aun más, que le lleve a triunfar en su vida profesional. Y es que como afirma Victor Küppers:

“( $\boldsymbol{V}=(\boldsymbol{c}+\boldsymbol{h}) \times \boldsymbol{A})$ Lo que vales es igual a la suma de tus conocimientos y tu experiencia o habilidad, pero lo que realmente es importante es la ACTITUD.

Mientras que conocimiento y experiencia suman, LA ACTITUD MULTIPLICA.”

\section{BIBLIOGRAFÍA}

Ali, Alisha, Hilary Catherine Murphy, and Sanjay Nadkarni. 2017. "Sustainable Development and Hospitality Education: Employers' Perspectives on the Relevance for Graduate Employability." Journal of Teaching in Travel and Tourism 17(3):159-72. Retrieved (https://doi.org/10.1080/15313220.2017.1331782).

Aneca. 2004. "Libro Blanco; Titulo de Grado En Turismo." Agencia Nacional de Evaluación de La Calidad y Acreditación 262. Retrieved (http://www.uned.es/espacio-europeo/pdf/Libro-blanco-turismo.pdf).

Anon. 2016. "Informe CYD2016 Graduados Universitarios y Mercado de Trabajao Capitulo 2." Retrieved (http://www.fundacioncyd.org/images/informeCyd/2016/Cap2_ICYD2016.pdf).

Boudjaoui, Mehdi, Jean Clénet, and Mokhtar Kaddouri. 2015. "La Formación En Alternancia En Francia: Entre Prácticas Sociales y Objeto de Investigación.” Educar 51(2):239-58. Retrieved (http://educar.uab.cat/article/view/679).

Canalis, Xavier. 2018. “¿Estudias Turismo? Esto Es Lo Que Más Valorarán de Ti Las Empresas.” Retrieved (https://www.hosteltur.com/127424_estudias-turismo-esto-es-lo-valoraran-ti-empresas.html\#).

Canalis, Xavier. 2014. "Empleo Turístico En España: ¿cantidad o Calidad?" Hosteltur. Retrieved (https://www.hosteltur.com/183680_empleo-turistico-espana-cantidad-calidad.html\#).

Caparr, Antonio. 2007. "La Naturaleza de La Temporalidad En El Empleo : Nueva Evidencia Empírica ( * )." 49:59-102.

Crosier, David, Anna Horvath, Viera Kerpanova, Daniela Kocanova, and Jari Riihelainen. 2006. "Modernización de La Educación Superior En Europa." 812.

Decreto, Real. 1996. "9296 50.” 14947-49.

El, Alfonso X., D. E. La, and Alfara Del. 2011. "Oferta de Titulaciones." (1):1-318.

España, Gobierno de. n.d. "Ministerio de Educación, Cultura y Deporte." Retrieved (http://www.mecd.gob.es/educacion-mecd/mc/lomce/lomce/presentacion.html).

Eurico, Sofia Teixeira, João Albino Matos Da Silva, and Patrícia Oom Do Valle. 2015. "A Model of Graduates Satisfaction and Loyalty in Tourism Higher Education: The Role of Employability." Journal of Hospitality, Leisure, Sport and Tourism Education 16:30-42. Retrieved (http://dx.doi.org/10.1016/j.jhlste.2014.07.002).

Exceltur. 2018. "Estudio Sobre Empleo En El Sector Turísticos Español."

Gómez, Venancio Bote. 2015. "Turismo y Desarrollo Económico En España: Del Insuficiente Reconocimiento a La Revalorización de Su Función Estratégica." Papers de Turisme (14-15):117-29. Retrieved (http://www.papersdeturisme.gva.es/ojs/index.php/Papers/article/view/420).

Hosteltur. 2018. "El Empleo En Turismo Repuntó Un 4,5\% En El Último Trimestre de 2017." Retrieved (https://www.hosteltur.com/126453_empleo-turismo-repunto-45-ultimo-trimestre-2017.html).

Hosteltur. n.d. "Las Empresas, En Busca de Talento Que Gestione La Transformación Digital." Retrieved (https://www.hosteltur.com/127907_empresas-busca-talento-gestione-transformacion-digital.html).

INE. 2015. "Situación Laboral a Finales De 2014." 2014:20. Retrieved (http://www.ine.es/prensa/np957.pdf).

Lauterbach, Uwe and Ute Lanzendorf. 1997. "El Sistema Dual de Formación Profesional En Alemania: Funcionamiento y Situación Actual." Revista Interuniversitaria de Formación Del Profesorado 30:51-68. Retrieved (http://maxconn.aufop.com/aufop/uploaded_files/articulos/1223244759.pdf).

Lluís, Jordi, Coiduras Rodríguez, Sofia Isus Barado, Isabel Del, and Arco Bravo. 2015. "Formación Inicial de Docentes En Alternancia. Análisis Desde Las Percepciones de Los Actores En Una Experiencia de Integración de Aprendizajes." 512:2014-8801. Retrieved (http://dx.doi.org/10.5565/rev/educar.670).

Lozano Francisco, Samara and Isabel Vázquez Bermúdez. 2014. Turismo y Empleo: Estudio de Una 
Zona Geográfica (Sevilla y Comparación Con Andalucia), Trabajo Fin de Grado : Grado En Turismo. [Sevilla : El Autor]. Retrieved March 15, 2018 (http://fama.us.es/record).

Mouillour, Isabelle Le. 2017. "Employment Aspects in Dual Training in Germany Education and Training System in Germany." (May).

OIT. 2016. "Perspectivas Sociales Empleo Mundo." Organizacion Internacional Del Trabajo 4. Retrieved (http://www.ilo.org/global/research/global-reports/youth/2016/WCMS_513747/lang--es/index.htm).

Roura, Juan Ramón Cuadrado. 1999. "El Sector Servicios y El Empleo En España: Evolución Reciente y Perspectivas de Futuro." Revista de Economía Aplicada VIII:177-80.

Sevilla, Universidad D. E. 1999. "Turismo , a Impartir En La Escuela Universitaria de Estudios Empresariales . La Junta de Gobierno de Esta Universidad, En Sesión Celebrada El Día 14 de Junio de 1999 , Aprobó El Plan de Estudios Conducente a La Obtención Del Título de Diplomado En Turism."

UNESCO, Cátedra. 2015. Barómetro de Empleabilidad y Empleo 2015 Barómetro de Empleabilidad y Empleo 2015. 